\title{
Expression pattern of Zinc finger protein 185 in mouse testis and its role in regulation of testosterone secretion
}

\author{
XINGUO YOU*, LU WEI*, SHUTONG FAN, WEIWEI YANG, XIAOYING LIU, \\ GUOHUI WANG, YI MAN, ZHIFANG PAN and WEIGUO FENG
}

College of Bioscience and Technology, Weifang Medical University, Weifang, Shandong 261053, P.R. China

Received December 10, 2016; Accepted May 31, 2017

DOI: $10.3892 / \mathrm{mmr} .2017 .6797$

\begin{abstract}
Zinc finger protein 185 (ZNF185) belongs to the ZNF family and is involved in cell proliferation and differentiation. To the best of our knowledge, the association between $\mathrm{ZNF} 185$ and male reproduction is unknown. In the present study, the expression and localization of ZNF185 in mouse testis, as well as its role in testosterone secretion, cell cycle progression and apoptosis of mouse Leydig cells were investigated. The results of the immunofluorescence analysis indicated that ZNF185 was highly expressed in Leydig cells of the mouse testis, and primarily localized in the cytoplasm. The results of quantitative polymerase chain reaction and western blot analyses further validated that ZNF185 expression was significantly higher in Leydig cells and sperm compared with that in Sertoli cells. Subsequently, the expression pattern of ZNF185 in mouse testis was determined at different developmental stages. The results demonstrated that the expression of ZNF185 was highest in the testis of 10-week-old mice and lowest in 2-week-old mice. Furthermore, the role of ZNF185 in Leydig cells of the mouse testis was investigated. Different concentrations of luteinizing hormone ( $\mathrm{LH}$ ) were used to stimulate the Leydig cells and subsequently the expression of ZNF185, and testosterone concentration was detected. The results revealed that LH upregulated the expression of ZNF185 and testosterone secretion, and ZNF185 expression was significantly positively correlated with testosterone secretion. To further validate whether ZNF185 was involved in testosterone secretion, lentiviral-mediated RNA interference was used to knock down ZNF185 expression in Leydig cells. The results demonstrated that ZNF185 expression and testosterone secretion of Leydig
\end{abstract}

Correspondence to: Dr Weiguo Feng or Dr Zhifang Pan, College of Bioscience and Technology, Weifang Medical University, 7166 Baotong Street, Weifang, Shandong 261053, P.R. China

E-mail: sdaqfwg@126.com

E-mail:pzhifang@163.com

*Contributed equally

Key words: zinc finger protein 185, Leydig cells, testis, mouse, testosterone cells were decreased significantly. In addition, the results demonstrated that the knockdown of ZNF185 expression did not significantly affect cell cycle progression or apoptosis. Taken together, the results of the present study revealed that ZNF185 was highly expressed in Leydig cells of the testis and involved in the secretion of testosterone. These results have contributed to the elucidation of the mechanism underlying male reproduction and may provide a novel target for the treatment of infertility, and the development of a contraceptive vaccine.

\section{Introduction}

Most of the testosterone in the male is synthesized by the testicular Leydig cells $(1,2)$, which is mainly involved in the male reproductive system, participating in the regulation of sexual differentiation, spermatogenesis, maintaining sexual behavior and promoting the development of the accessory sex gland (3-5). The recent study shows that the testosterone secretion by Leydig cells is regulated by multiple genes (6-8). Therefore, identification of novel regulatory genes is important for elucidating the regulation mechanism of testosterone secretion in Leydig cells and spermatogenesis in testis.

Zinc finger protein is one kind of transcription factor, which is widely existed in organisms, and plays an important role in cell proliferation, cell differentiation and cell apoptosis (9-12). At present, several studies demonstrated that zinc finger protein was expressed specifically in testis, such as ZNF230 (13), ZNF105 (14) and ZNF300 (15), and closely associated with spermatogenesis and male reproduction.

ZNF185, also known as ZFP185, is belonging to the ZNF family and identified by Heiss for the first time in 1997 (16). ZNF185 is located on DXS52 region of the long arm of chromosome Xq28 (16), with a LIM zinc-binding domain at the $\mathrm{C}$ terminus and an actin-targeting domain at the $\mathrm{N}$ terminus (17). The previous study indicates that ZNF185 is involved in cell proliferation and cell differentiation $(18,19)$. However, the relationship between ZNF185 and male reproduction is unknown.

In this study, we investigated the expression and localization of ZNF185 in mouse testis by qPCR, western blotting and immunofluorescence. Finally, we studied the effects of ZNF185 on testosterone secretion, cell cycle and cell apoptosis by lentiviral mediated RNA interference. 


\section{Materials and methods}

Animals and tissue preparation. Thirty ICR male mice (2-week, 10-week and 60-week-old; $\mathrm{n}=10$ in each group) were purchased from Weifang Medical University Animal Center (Weifang, China). The mice were sacrificed to obtain testis tissue and sperm used for following study. Animal care and experimental procedures were carried out according to the Animal Research Committee guidelines of Weifang Medical University. The protocol was approved by the Ethics Committee of Animal of Shandong (permit no. 20100326).

The isolation and culture of Leydig cell and Sertoli cell. The isolation and culture of Leydig cell was performed as previous study (20). Briefly, the mouse testes were transferred to a centrifuge tube containing $0.75 \mathrm{mg} / \mathrm{ml}$ collagenase IV (Sigma, St. Louis MO, USA) and digested for $20 \mathrm{~min}$ at $37^{\circ} \mathrm{C}$. After termination of digestion, the supernatant was centrifuged at $200 \mathrm{~g}$ for $5 \mathrm{~min}$. The supernatant was discarded, and then the pellet was resuspended in Dulbecco's modified Eagle's medium (DMEM; Invitrogen, Shanghai, China) supplemented $10 \%$ fetal bovine serum (FBS; Gibco, Shanghai, China) at $37^{\circ} \mathrm{C}$ and $5 \% \mathrm{CO}_{2}$.

The isolation and culture of Sertoli cell was performed as previous study (21). In brief, the seminiferous tubules of testes were washed by phosphate-buffered saline (PBS) and incubated using $3 \mathrm{mg} / \mathrm{ml}$ collagenase I for $20 \mathrm{~min}$ at $37^{\circ} \mathrm{C}$ on the shaker, and then washed three times using DMEM medium. After washing three times, the samples were further digested using $3 \mathrm{mg} / \mathrm{ml}$ collagenase I, $1 \mathrm{mg} / \mathrm{ml}$ trypsin (Sigma) and $2 \mathrm{mg} / \mathrm{ml}$ hyaluronidase (Sigma) for $20 \mathrm{~min}$ at $37^{\circ} \mathrm{C}$. Finally, the pellet was resuspended and transferred into DMEM supplemented $10 \%$ fetal bovine serum and incubated at $37^{\circ} \mathrm{C}$ and $5 \% \mathrm{CO}_{2}$.

Immunofluorescence. Immunofluorescence studies were performed on mouse testis sections and cells (Leydig cells, Sertoli cells and sperm) using anti-ZNF185 antibodies (Santa Cruz Biotechnology, Inc., Dallas, TX, USA), as described previous study (22).

$q P C R$. The mRNA level of ZNF185 expression in mouse testis was detected by qPCR using SYBR Premix Ex Taq kit (Takara Bio, Dalian, China). The primer sequence of ZNF185 was as follows: Forward, 5'-CTCCCAGCATCGCCCCTCTAAG-3'; reverse, 5'-GCCTGGGACCTCCGTTTCTGCT-3'. The primer sequence of $\beta$-actin was as follows: Forward, 5'-CGTTGA CATCCGTAAAGACC-3'; reverse, 5'-ACAGTCCGCCTA GAAGCAC-3'. The total volume of reaction mixture is $20 \mu \mathrm{l}$, containing $2 \mu \mathrm{l}$ of cDNA, $0.4 \mu \mathrm{l}$ Dye, $0.6 \mu \mathrm{l}$ of each primer, $10 \mu \mathrm{l} \mathrm{SYBR}$ and $6.4 \mu 1 \mathrm{H}_{2} \mathrm{O}$. Each reaction was performed in triplicate. The data was analyzed by the $2^{-\Delta \Delta C q}$ method (23).

Western blotting. Mouse testis tissue and cells were homogenized and treated by lysis buffer kit (Beyotime Institute of Biotechnology, Shanghai, China). The concentration of protein was determined using BCA assay kit (Beyotime Institute of Biotechnology). The proteins were subjected to SDS-PAGE and then transferred to the PVDF membrane. The membrane was blocked by $5 \%$ milk and incubated overnight with anti-ZNF185 and anti- $\beta$-actin (Beyotime Institute of Biotechnology) at $4^{\circ} \mathrm{C}$.
After washing by PBST, the membrane was incubated with the secondary antibody (Beyotime Institute of Biotechnology) for $1.5 \mathrm{~h}$ at room temperature. Finally, the reaction bands were detected using the enhanced chemiluminescence reaction kit (ECL; Beyotime Institute of Biotechnology).

Cell preparation. To investigate the relationship between ZNF185 and testosterone secretion, different concentrations of LH were used to stimulate the testosterone secretion of Leydig cells. After the addition of LH, Leydig cells were cultured for $24 \mathrm{~h}$, and then the supernatants were collected for testosterone assay and the cells were collected to detect the expression of ZNF185.

To further study the role of ZNF185 in regulation of testosterone secretion, the lentiviral mediated RNA interference targeting ZNF185 was constructed by Sangon Biotech (Shanghai, China), which was transfected into Leydig cells to knockdown the ZNF185 expression.

Testosterone measurement. To determine the effect of ZNF185 knockdown on testosterone secretion in Leydig cells, the cells were cultured for $48 \mathrm{~h}$. The cells were trypsinized and centrifuged at $500 \mathrm{~g}$ for $10 \mathrm{~min}$. Subsequently, the culture supernatants were collected for testosterone analysis using the testosterone test kit (Mlbio, Shanghai, China) according to the manufacturer's instructions.

Cell cycle and cell apoptosis analysis. Leydig cells were harvested and centrifuged at $200 \mathrm{~g}$ for $15 \mathrm{~min}$. After washing by PBS, the cells were fixed by $70 \%$ ethanol. Subsequently, the cells were incubated in PBS with propidium iodide (PI, $5 \mu \mathrm{g} / \mathrm{ml})$ and RNase $(200 \mu \mathrm{g} / \mathrm{ml})$ for $30 \mathrm{~min}$ at room temperature. Finally, the cell cycle was assessed by flow cytometric analysis.

Leydig cells were collected and centrifuged at $200 \mathrm{~g}$ for $15 \mathrm{~min}$. After removing the supernatant, the cells were washed by ice-cold PBS. The cells were then resuspended in binding buffer, and incubated in binding buffer containing PI $(5 \mu \mathrm{g} / \mathrm{ml})$ and Annexin V $(5 \mu \mathrm{g} / \mathrm{ml})$ for $30 \mathrm{~min}$ at room temperature. Ultimately, the cell apoptosis were assessed by flow cytometric analysis, according to the manufacturer's instructions.

Statistical analysis. Statistical comparisons were evaluated by an independent sample t-test. Data were expressed as mean $\pm \mathrm{SE}$, and $\mathrm{P}<0.05$ was considered to indicate a statistically significant difference. The SPSS software version 19.0 (SPSS, Inc., Chicago, IL, USA) was used for analysis.

\section{Results}

The localization and expression of ZNF185 in the testis of mouse. The localization of ZNF185 in the testis of mouse was detected by immunofluorescence (Fig. 1). The in vivo experimental results showed that ZNF185 was present in Leydig cell, Sertoli cell and sperm. Nevertheless, the expression of ZNF185 in Leydig cell and sperm was significantly higher than Sertoli cell $(\mathrm{P}<0.05$, Fig. $1 \mathrm{~A})$. The in vitro experimental results showed that ZNF185 was mainly localized in the cytoplasm of Leydig cell and Sertoli cell, as well as the head and tail of the sperm (Fig. 1C). 

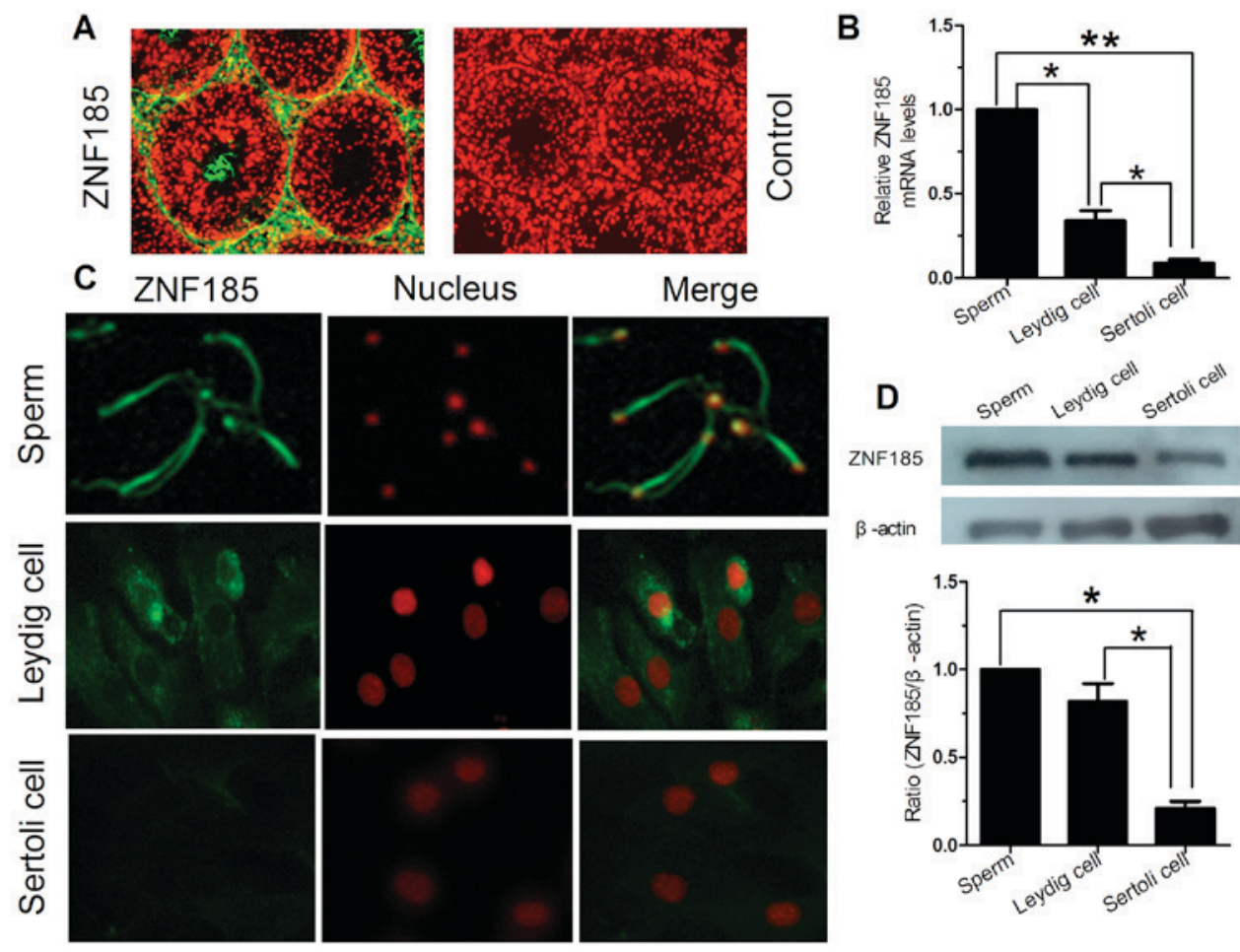

Figure 1. The localization and expression of ZNF185 in mouse testis. (A) Localization of ZNF185 in testis tissue was detected by immunofluorescence. ZNF185 was represented as green. Nucleus was stained red with PI. Control: primary antibody was replaced by rabbit IgG. Magnification, 200x. (B) The relative mRNA of ZNF185 was monitored by quantitative polymerase chain reaction. (C) Localization of ZNF185 in Leydig cells, Sertoli cells and sperm was detected by immunofluorescence. ZNF185 was represented as green. Nucleus was stained red with PI. Magnification, 400x. (D) The protein expression of ZNF185 was detected by western blot analysis. The results are representative of three independent experiments, and performed in triplicate. ${ }^{*} \mathrm{P}<0.05$, ${ }^{* *} \mathrm{P}<0.01$. Error bars indicate standard error. PI, propidium iodide; ZNF185, zinc finger protein 185.

Subsequently, the expression of ZNF185 in Leydig cell, Sertoli cell and sperm was studied by real-time PCR and western blot. The PCR results showed that the expression of ZNF185 in sperm was significantly higher than Leydig cell and Sertoli cell $(\mathrm{P}<0.05)$, while the expression of ZNF185 in Leydig cell was significantly lower than sperm, but higher than Sertoli cell ( $\mathrm{P}<0.05$, Fig. 1B). Western blot was used to further validate the expression of ZNF185. As shown in Fig. 1D, the protein expression level of ZNF185 was significantly higher in Leydig cell and sperm compared with Sertoli cell $(\mathrm{P}<0.05)$, and there was no significant difference between them $(\mathrm{P}>0.05)$.

The expression of ZNF185 in different developmental stages of mouse testis. The ZNF185 expression in mouse testis aged 2 weeks, 10 weeks and 60 weeks was evaluated by real-time PCR and western blot. The results showed that ZNF185 was expressed in all detected time points, and the protein expression pattern of ZNF185 was similar to the mRNA expression, with age-dependent manner (Fig. 2). The expression of ZNF185 in the testis of 10 week old mouse was significantly higher than 2 week old group and 60 week old group $(\mathrm{P}<0.05)$. The expression of ZNF185 in the testis of 60 week old mouse was lower than 10 week old group $(\mathrm{P}>0.05)$, while significantly higher than 2 week old group $(\mathrm{P}<0.05)$.

LH treatment up-regulated the ZNF185 expression and testosterone secretion. Using different concentrations of LH to stimulate the Leydig cell, subsequently, the ZNF185 expression was studied by real-time PCR and western blot, and the testosterone concentration was detected by ELISA. The results showed that $\mathrm{LH}$ could up-regulate the expression of ZNF185 and testosterone secretion, with concentration-dependent manner (Fig. 3). With the increase of LH concentration, the expression of ZNF185 and testosterone secretion was also elevated. Compared with the control group, the expression of ZNF185 and testosterone secretion in $0.01 \mathrm{IU} / \mathrm{ml}$ group was significantly higher $(\mathrm{P}<0.05)$. Further statistical analysis showed that ZNF185 expression was significantly positively correlated with testosterone secretion $\left(r^{2}=0.92, P<0.05\right)$.

Lentiviral mediated RNA interference inhibited the ZNF185 expression and testosterone secretion. In order to further study the role of ZNF185 in testosterone secretion, the lentiviral mediated RNA interference targeting ZNF185 was constructed (Fig. 4). The Leydig cells of testis were transfected by the lentivirus, subsequently, the ZNF185 expression was investigated by qPCR and western blotting. The results showed that the lentivirus could significantly inhibit the ZNF185 expression in mRNA and protein level compared to the control $(\mathrm{P}<0.05$, Fig. 4A and $\mathrm{C})$.

After transfection by the lentivirus, the testosterone secretion level in Leydig cells was detected by ELISA. The results showed that the testosterone secretion level in the lentiviral mediated RNA interference group was significantly lower than the control group $(\mathrm{P}<0.05$, Fig. $4 \mathrm{~B})$. This result indicated that ZNF185 was closely associated with the testosterone secretion. 

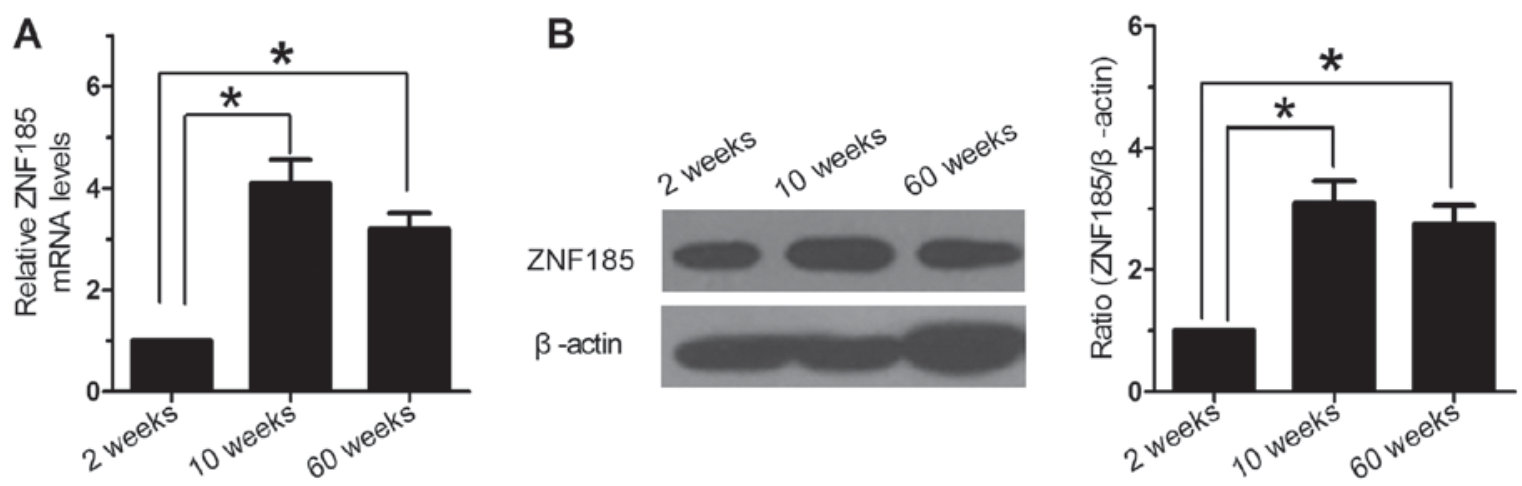

Figure 2. Developmental expression model of ZNF185 in postnatal mouse testis. (A) The mRNA level of ZNF185 at different developmental stages was monitored by quantitative polymerase chain reaction. (B) The protein level of ZNF185 at different developmental stages was detected by western blotting. The results are representative of three independent experiments, and performed in triplicate. " $\mathrm{P}<0.05$. Error bars indicate standard error. ZNF185, zinc finger protein 185.

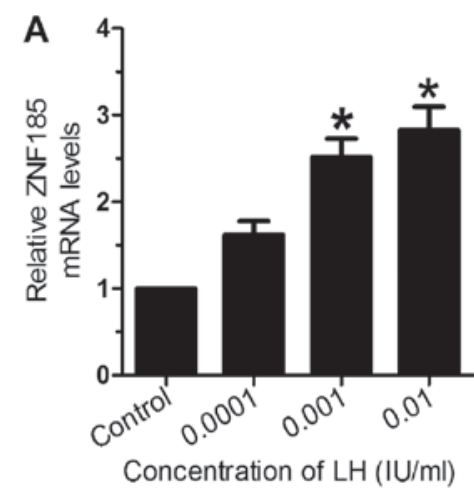

C

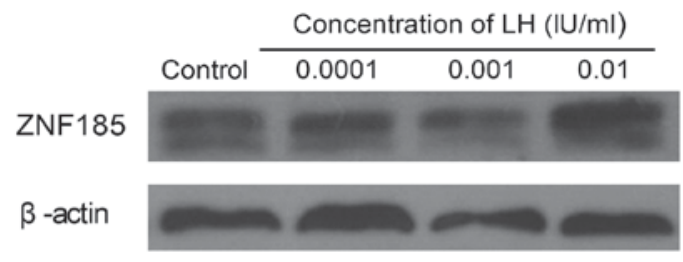

B
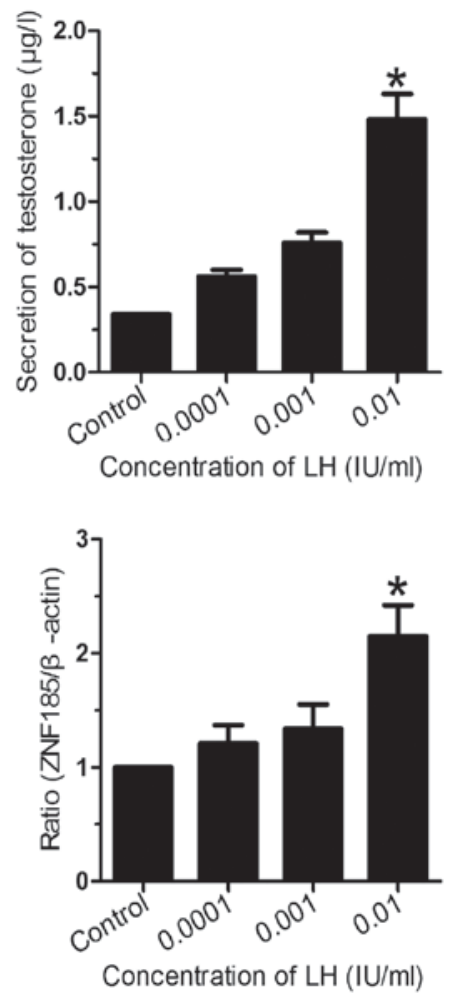

Figure 3. LH increased ZNF185 expression and testosterone secretion. Leydig cells were treated with different concentrations of LH. (A) The mRNA level of ZNF185 was monitored by quantitative polymerase chain reaction. (B) The testosterone was detected by the immunoassay kit. (C) The protein level of ZNF185 was monitored by western blotting. The results are representative of three independent experiments, and performed in triplicate. ${ }^{*} \mathrm{P}<0.05$. Error bars indicate standard error. ZNF185, zinc finger protein 185; LH, luteinizing hormone .

Knockdown of ZNF185 expression did not affect cell cycle and cell apoptosis of Leydig cells. After transfection by the lentivirus, the cell cycle and cell apoptosis were tested by flow cytometry. The results showed that knockdown of ZNF185 expression did not significantly affect cell cycle and cell apoptosis compared to the control ( $\mathrm{P}>0.05$, Fig. 5).

\section{Discussion}

Recently, studies demonstrated that several zinc finger proteins including ZNF230 (13), ZNF105 (14) and ZNF300 (15) were expressed specifically in testis and closely related to spermatogenesis and male reproduction. ZNF185 also belongs to the zinc finger protein family. However, the role of ZNF185 in male reproduction is unknown.

In our present study, we demonstrated that ZNF185 was highly expressed in Leydig cells of the mouse testis and involved in the secretion of testosterone for the first time. This conclusion was based on the results of molecular and immunological experiments. The results of immunofluorescence indicated that ZNF185 was highly expressed in Leydig cells of the mouse testis in vivo. The results of real-time PCR and western blot further validated that ZNF185 expressed significantly higher in Leydig cells and sperm compared with Sertoli cells in vitro $(\mathrm{P}<0.05)$. 

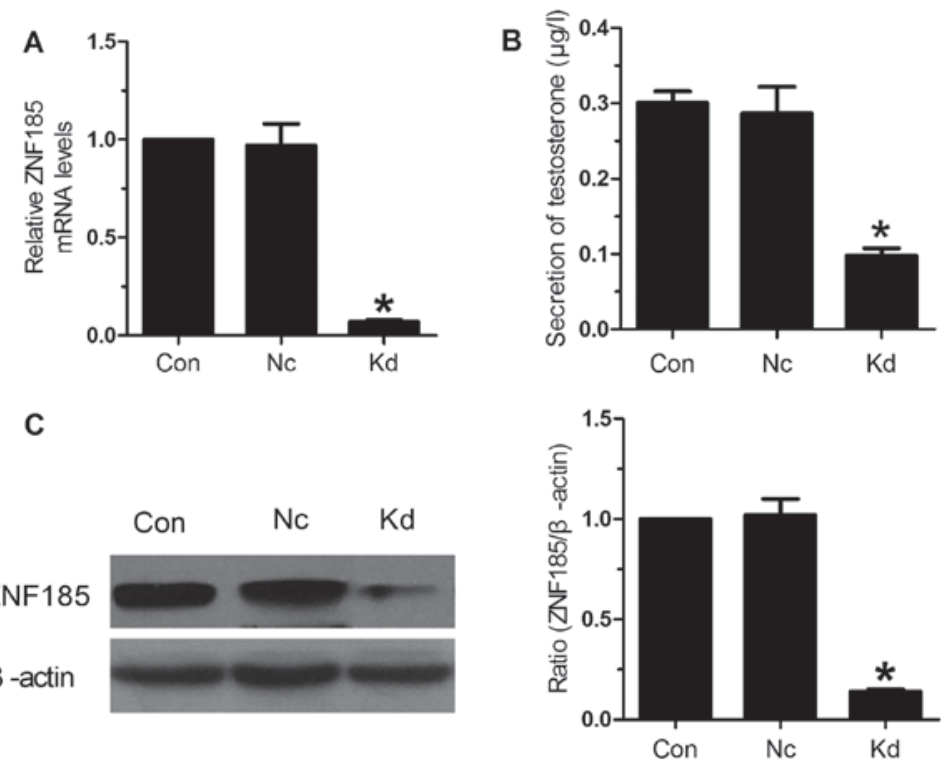

Figure 4. Lentivirus-ZNF185-shRNA inhibited the ZNF185 expression and testosterone secretion in Leydig cells. Leydig cells were cultured for $48 \mathrm{~h}$ after lentivirus transfection. (A) The mRNA level of ZNF185 was monitored by qPCR. (B) The testosterone was detected by the immunoassay kit. (C) The protein level of ZNF185 was monitored by western blotting. Con group, control Leydig cells; NC group, Leydig cells transduced using negative control lentivirus; Kd group, Leydig cells transduced using lentivirus-ZNF185-shRNA. The results are representative of three independent experiments, and performed in triplicate. ${ }^{*} \mathrm{P}<0.05$. Error bars indicate standard error. ZNF185, zinc finger protein 185; sh, short hairpin.

A

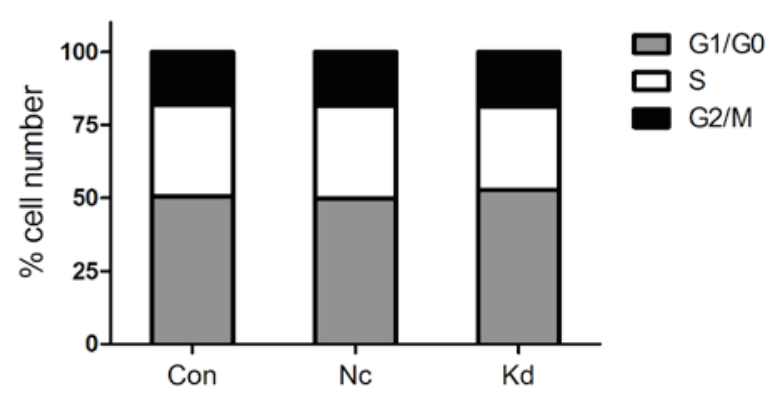

B

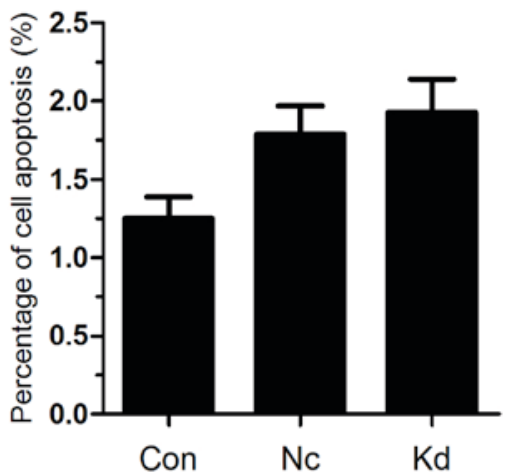

Figure 5. Downregulated ZNF185 exhibited no effect on cell cycle and cell apoptosis of Leydig cells. (A) Cell cycle of different Leydig cell groups. (B) Cell apoptosis of different Leydig cell groups. Con group, control Leydig cells; NC group, Leydig cells transduced using negative control lentivirus; Kd group, Leydig cells transduced using lentivirus-ZNF185-shRNA. The results are representative of three independent experiments, and performed in triplicate. Error bars indicate standard error. ZNF185, zinc finger protein 185; sh, short hairpin.

Furthermore, the results of immunofluorescence showed that ZNF185 was mainly localized in the cytoplasm of Leydig cells and Sertoli cells.

Previous studies have indicated that, in order to satisfy the physiological functions of different developmental stages, the testis show stage-specific gene expression pattern (24-26). Consequently, we investigated the expression pattern of ZNF185 in mouse testis at different developmental stages in this study. The results showed that ZNF185 was expressed with age-dependent manner. The expression of ZNF185 was highest in the testis of 10 week old mouse. The expression of ZNF185 in the testis of 60 week old mouse was lower than 10-week-old group ( $\mathrm{P}>0.05)$, while significantly higher than 2 -week-old group $(\mathrm{P}<0.05)$. Taken together, the current results suggested that ZNF185 plays an important role in male reproduction.

To verify whether there was a relationship between ZNF185 and male reproduction, we used different concentrations of $\mathrm{LH}$ to deal with Leydig cells, and then detect the expression of ZNF185 and testosterone concentration. The results revealed that LH could up-regulate the expression of ZNF185 and testosterone secretion. Accompanying with the increase of LH concentration, the expression of ZNF185 and testosterone secretion was raised. Further statistical analysis showed that ZNF185 expression was significantly positively correlated with testosterone secretion $\left(\mathrm{r}^{2}=0.92, \mathrm{P}<0.05\right)$.

It is well known that most of the testosterone in the male is synthesized by the testicular Leydig cells and contribute to the development of Sertoli cells and germ cells (26). Accordingly, Leydig cells are essential for maintaining normal reproductive activity and the testosterone is involved in the regulation of sexual differentiation, spermatogenesis, maintaining sexual behavior and promoting the development of the accessory sex gland $(3-5,27)$. The testosterone secretion of Leydig cells is regulated by various factors which involved in endocrine and 
paracrine signaling, and these factors are crucial for reproductive activity (26). Therefore, a variety of specific genes of Leydig cells have been identified as pivotal regulatory factors in the process of reproduction (6-8). In this study, the results demonstrated that ZNF185 was highly expressed in Leydig cells of the testis and expressed highest in adult mouse. In addition, we also found that ZNF185 expression pattern was significantly positively correlated with testosterone secretion. As a result, we speculated that ZNF185 was critical for testosterone secretion of Leydig cells.

To further validate whether ZNF185 involved in testosterone secretion, the lentiviral mediated RNA interference was used to knock down the ZNF185 expression in Leydig cells, and then the testosterone secretion was detected. The results showed that the lentivirus mediated RNA interference could significantly inhibit the ZNF185 expression in mRNA and protein level, meanwhile, the testosterone secretion of Leydig cells was decreased obviously. These results demonstrated that ZNF185 was directly involved in testosterone secretion of Leydig cells. However, the mechanism of testosterone secretion in Leydig cells regulated by ZNF185 need to be further studied.

In addition, we investigated the cell biological function, and the results showed that knockdown of ZNF185 expression did not significantly affect cell cycle and cell apoptosis. These results suggested that ZNF185 was not related to the cell cycle and cell apoptosis. Nevertheless, whether ZNF185 involved in other cell biological function need to be explored.

In conclusion, we demonstrated that ZNF185 was highly expressed in Leydig cells of the testis and involved in the secretion of testosterone for the first time. Our results contributed to elucidation of the mechanism of male reproduction, and might provide a target for the treatment of infertility and the development of contraceptive vaccine.

\section{Acknowledgements}

The study was supported by the Natural Science Foundation of Shandong Province (nos. ZR2013CM032 and ZR2014CL034), the Science and Technology Development Plan of Shandong Province (no. 2015GSF118178) and Project of Health and Family Planning Commission of Shandong Province (no. 201411).

\section{References}

1. Alexandrova ML and Bochev PG: Reduced extracellular phagocyte oxidative activity, antioxidant level changes and increased oxidative damage in healthy human blood as a function of age. Age 31: 99-107, 2009.

2. Chen H, Cangello D, Benson S, Folmer J, Zhu H, Trush MA and Zirkin BR: Age-related increase in mitochondrial superoxide generation in the testosterone-producing cells of Brown Norway rat testes: Relationship to reduced steroidogenic function? Exp Gerontol 36: 1361-1373, 2001.

3. Sharpe RM, Maddocks S, Millar M, Kerr JB, Saunders PTK and Mckinnell C: Testosterone and spermatogenesis identification of stage-specific, androgen-regulated proteins secreted by adult rat seminiferous tubules. J Androl 13: 172-184, 1992.

4. Zirkin BR and Tenover JL: Aging and declining testosterone: Past, present, and hopes for the future. J Androl 33: 1111-1118, 2012.

5. Walker WH: Molecular mechanisms of testosterone action in spermatogenesis. Steroids 74: 602-607, 2009.

6. Ahn SW, Gang GT, Kim YD, Ahn RS, Harris RA, Lee CH and Choi HS: Insulin directly regulates steroidogenesis via induction of the orphan nuclear receptor DAX-1 in testicular Leydig cells. J Bio Chem 288: 15937-15946, 2013.
7. Matzkin ME, Yamashita S and Ascoli M: The ERK1/2 pathway regulates testosterone synthesis by coordinately regulating the expression of steroidogenic genes in Leydig cells. Mol Cell Endocrinol 370: 130-137, 2013

8. Mishra J, Gautam M, Dadhich R, Kowtharapu BS and Majumdar SS: Peritubular cells may modulate Leydig cell-mediated testosterone production through a nonclassic pathway. Fertil Steril 98: 1308-1317. e1, 2012.

9. Ma J, Mi C, Wang KS, Lee JJ and Jin X: Zinc finger protein 91 (ZFP91) activates HIF-1 $\alpha$ via NF- $\mathrm{kB} / \mathrm{p} 65$ to promote proliferation and tumorigenesis of colon cancer. Oncotarget 7: 36551-36562, 2016.

10. Tseng KY and Lin S: Zinc finger factor 521 enhances adipogenic differentiation of mouse multipotent cells and human bone marrow mesenchymal stem cells. Oncotarget 6: 14874-14884, 2015.

11. Yang F, Ma H, Feng L, Lian M, Wang R, Fan E and Fang J: Zinc finger protein $\mathrm{x}$-linked (ZFX) contributes to patient prognosis, cell proliferation and apoptosis in human laryngeal squamous cell carcinoma. Int J Clin Exp Pathol 8: 13886-13899, 2015.

12. Buchner DA, Charrier A, Srinivasan E, Wang L, Paulsen MT, Ljungman M, Bridges D and Saltiel AR: Zinc finger protein 407 (ZFP407) regulates insulin-stimulated glucose uptake and glucose transporter 4 (Glut4) mRNA. J Biol Chem 290: 6376-6386, 2015.

13. Zhang S, Qiu W, Wu H, Zhang G, Huang M, Xiao C, Yang J, Kamp C, Huang X, Huellen K, et al: The shorter zinc finger protein ZNF230 gene message is transcribed in fertile male testes and may be related to human spermatogenesis. Biochem J 359: 721-727, 2001.

14. Zhou H, Liu LH, Zhang H, Lei Z and Lan ZJ: Expression of zinc finger protein 105 in the testis and its role in male fertility. Mol Reprod Dev 77: 511-520, 2010.

15. Cao Y, Li JX, Ji CN, Xu XW and Wu M: Molecular cloning and characterization of a novel splice variant of human ZNF300 gene, which expressed highly in testis. DNA Seq 18: 312-315, 2007.

16. Heiss NS, Gloeckner G, Bächner D, Kioschis P, Klauck SM, Hinzmann B, Rosenthal A, Herman GE and Poustka A: Genomic structure of a novel LIM domain gene (ZNF185) in Xq28 and comparisons with the orthologous murine transcript. Genomics 43: 329-338, 1997.

17. Furukawa D, Chijiwa T, Matsuyama M, Mukai M, Matsuo EI, Nishimura O, Kawai K, Suemizu H, Hiraoka N, Nakagohri T, et al: Zinc finger protein 185 is a liver metastasis-associated factor in colon cancer patients. Mol Clin Oncol 2: 709-713, 2014.

18. Vanaja DK, Cheville JC, Iturria SJ and Young CY: Transcriptional silencing of zinc finger protein 185 identified by expression profiling is associated with prostate cancer progression. Cancer Res 63: 3877-3882, 2003.

19. Zhang J, Gong A and Young C: ZNF185, an actin-cytoskeleton-associated growth inhibitory LIM protein in prostate cancer. Oncogene 26: 111-122, 2007.

20. Zhong L, Sun J, Liu GH, Zhu YJ and Zhu J: Research on the steroidogenesis of proliferated Leydig cells in vitro. J Artif Organs 16: 229-233, 2013.

21. Jiang X, Zhang H, Shi Y, Yin S, Zhang Y, Yang W, Zheng W, Wang L, Wang Z, Bukhari I, et al: Specific deficiency of Plzf paralog,Zbtb20, in Sertoli cells does not affect spermatogenesis and fertility in mice. Sci Rep 4: 7062, 2014.

22. Li Y, Sosnik J, Brassard L, Reese M, Spiridonov NA, Bates TC, Johnson GR, Anguita J, Visconti PE and Salicioni AM: Expression and localization of five members of the testis-specific serine kinase (Tssk) family in mouse and human sperm and testis. Mol Hum Reprod 17: 42-56, 2011.

23. Livak KJ and Schmittgen TD: Analysis of relative gene expression data using real-time quantitative PCR and the 2(-Delta Delta(T)) method. Methods 25: 402-408, 2001.

24. Johnston DS, Olivas E, DiCandeloro P and Wright WW: Stage-specific changes in GDNF expression by rat Sertoli cells: A possible regulator of the replication and differentiation of stem spermatogonia. Biol Reprod 85: 763-769, 2011.

25. Johnston DS, Wright WW, DiCandeloro P, Wilson E, Kopf GS and Jelinsky SA: Stage-specific gene expression is a fundamental characteristic of rat spermatogenic cells and Sertoli cells. Proc Natl Acad Sci USA 105: 8315-8320, 2008.

26. Yang Q, Hao J, He M, Chen M and Li G: Localization and expression patterns of prolactin-like protein $\mathrm{J}$ in mouse testis. Mol Med Rep 10: 255-261, 2014.

27. Midzak AS, Chen H, Papadopoulos V and Zirkin BR: Leydig cell aging and the mechanisms of reduced testosterone synthesis. Mol Cell Endocrinol 299: 23-31, 2009. 